\title{
Overexpression of fucosyltransferase 8 reverses the inhibitory effect of high-dose dexamethasone on osteogenic response of MC3T3-E1 preosteoblasts
}

\author{
Zhiming Wu ${ }^{1,2,3,4}$, Tianye Lin ${ }^{1,2,3}$, Pan Kang ${ }^{1,2,3}$, Zhikun Zhuang ${ }^{5}$, Haibin Wang ${ }^{1,2,3}$, Wei He ${ }^{1,6}$, Qiushi Wei ${ }^{1,6}$, Ziqi \\ Li ${ }^{\text {Corresp. } 1,6}$ \\ ${ }^{1}$ Guangzhou University of Chinese Medicine, Guangzhou, China \\ 2 Department of Joint Orthopaedic, The First Affiliated Hospital of Guangzhou University of Chinese Medicine, Guangzhou, China \\ 3 Lingnan Medical Research Center of Guangzhou University of Chinese Medicine, Guangzhou, China \\ 4 Shenzhen Hospital (Futian) of Guangzhou University of Chinese Medicine, Shenzhen City, Guangdong Province, China \\ 5 Department of Joint Orthopaedic, Quanzhou Orthopedic-Traumatological Hospital of Fujian Traditional Chinese Medicine University, Quanzhou, China \\ 6 Department of Joint Orthopaedic, The Third Affiliated Hospital, Guangzhou University of Chinese Medicine, Guangzhou, China \\ Corresponding Author: Ziqi Li \\ Email address: ionsev0214@126.com
}

Background: Core fucosylation catalyzed by FUT8 is essential for TGF- $\beta$ binding to TGF- $\beta$ receptors. Methods: Indirect TGF- $\beta 1$ binding assay was used to evaluate the ability of TGF- $\beta 1$ to bind to TGFBRs, Alizarin red and alkaline phosphatase staining were used to detect osteogenic differentiation and mineralization ability, western blot and quantitative RT-PCR were used to measure the differential expression of osteogenesis-related proteins and genes. Plasmid-mediated gain-of-function study. The scale of core fucosylation modification was detected by Lectin-blot and LCA laser confocal. Results: Our results showed that compared with vehicle treatment, high-dose $\left(10^{-6}\right.$ and $\left.10^{-5} \mathrm{M}\right)$ dexamethasone significantly inhibited cell proliferation, osteogenic differentiation, and FUT8 mRNA expression while promoting mRNA expression of adipogenesis-related genes in MC3T3-E1 cells, suggesting that downregulation of FUT8 is involved in the inhibitory effect of highdose dexamethasone on osteogenesis. Overexpression of FUT8 significantly promoted osteogenic differentiation and activated TGF- $\beta / S$ mad signaling in MC3T3-E1 cells in the presence of high-dose dexamethasone, suggesting that FUT8 reverses the inhibitory effect of high-dose dexamethasone on osteogenesis. In addition, lectin fluorescent staining and blotting showed that overexpression of FUT8 significantly reversed the inhibitory effects of high-dose dexamethasone on core fucosylation of TGFBR1 and TGFBR2. Furthermore, indirect TGF- $\beta 1$ binding assay showed that overexpression of FUT8 remarkably promoted TGF- $\beta 1$ binding to TGFBRs in MC3T3-E1 cells in the presence of high-dose dexamethasone.

Conclusions: Taken together, these results suggest that overexpression of FUT8 facilitates counteracting the inhibitory effect of dexamethasone on TGF- $\beta$ signaling and
Peer] reviewing PDF (2021:06:63083:1:0:NEW 19 Sep 2021) 
osteogenesis. 
1 Overexpression of fucosyltransferase 8 reverses the inhibitory effect of high-dose

2 dexamethasone on osteogenic response of MC3T3-E1 preosteoblasts

3 Running title: FUT8 pushes dexamethasone-osteogenesis

4

5 Zhiming $\mathrm{Wu}^{1,2,3,4}$, Tianye Lin ${ }^{1,2,3}$, Pan Kang ${ }^{1,2,3}$, Zhikun Zhuang ${ }^{5}$, Haibin Wang ${ }^{1,2,3}$, Wei He ${ }^{1,6}$,

6 Qiushi Wei ${ }^{1,6}$, Ziqi Li ${ }^{1,6^{*}}$

$7 \quad{ }^{1}$ Guangzhou University of Chinese Medicine, Guangzhou, 510405, China

8 2Department of Joint Orthopaedic, The First Affiliated Hospital of Guangzhou University of

9 Chinese Medicine, Guangzhou, 510405, China

$10{ }^{3}$ Lingnan Medical Research Center of Guangzhou University of Chinese Medicine, Guangzhou,

11510405 , China

$12{ }^{4}$ Shenzhen Hospital (Futian) of Guangzhou University of Chinese Medicine, No. 6001, North

13 Ring Road, Futian District,Shenzhen City, Guangdong Province, 518048, China

$14{ }^{5}$ Department of Joint Orthopaedic, Quanzhou Orthopedic-Traumatological Hospital of Fujian

15 Traditional Chinese Medicine University, Quanzhou, 362000, China

$16{ }^{6}$ Department of Joint Orthopaedic, The Third Affiliated Hospital, Guangzhou University of

17 Chinese Medicine, Guangzhou, 510378, China

18 *Corresponding author:

19 Ziqi Li

20 Guangzhou University of Chinese Medicine, Guangzhou, 510405, China; Department of Joint

21 Orthopaedic, The Third Affiliated Hospital, Guangzhou University of Chinese Medicine,

22 Guangzhou, 510378, China; Institude of Orthopedics, Guangzhou University of Chinese

23 Medicine, Guangzhou, 510378, China. 
24 E-mail: ionsev0214@126.com

\section{Abstract}

26 Background: Core fucosylation catalyzed by FUT8 is essential for TGF- $\beta$ binding to TGF- $\beta$

27 receptors.

28 Methods: Indirect TGF- $\beta 1$ binding assay was used to evaluate the ability of TGF- $\beta 1$ to bind to

29 TGFBRs, Alizarin red and alkaline phosphatase staining were used to detect osteogenic

30 differentiation and mineralization ability, western blot and quantitative RT-PCR were used to

31 measure the differential expression of osteogenesis-related proteins and genes. Plasmid-mediated

32 gain-of-function study. The scale of core fucosylation modification was detected by Lectin-blot

33 and LCA laser confocal.

34 Results: Our results showed that compared with vehicle treatment, high-dose $\left(10^{-6}\right.$ and $\left.10^{-5} \mathrm{M}\right)$

35 dexamethasone significantly inhibited cell proliferation, osteogenic differentiation, and FUT8

36 mRNA expression while promoting mRNA expression of adipogenesis-related genes in MC3T3-

37 E1 cells, suggesting that downregulation of FUT8 is involved in the inhibitory effect of high-

38 dose dexamethasone on osteogenesis. Overexpression of FUT8 significantly promoted

39 osteogenic differentiation and activated TGF- $\beta /$ Smad signaling in MC3T3-E1 cells in the

40 presence of high-dose dexamethasone, suggesting that FUT8 reverses the inhibitory effect of

41 high-dose dexamethasone on osteogenesis. In addition, lectin fluorescent staining and blotting

42 showed that overexpression of FUT8 significantly reversed the inhibitory effects of high-dose

43 dexamethasone on core fucosylation of TGFBR1 and TGFBR2. Furthermore, indirect TGF- $\beta 1$

44 binding assay showed that overexpression of FUT8 remarkably promoted TGF- $\beta 1$ binding to

45 TGFBRs in MC3T3-E1 cells in the presence of high-dose dexamethasone.

46 Conclusions: Taken together, these results suggest that overexpression of FUT8 facilitates 
47 counteracting the inhibitory effect of dexamethasone on TGF- $\beta$ signaling and osteogenesis.

48

49 Keywords: Dexamethasone; osteogenesis; fucosyltransferase 8; transforming growth factor beta 50 


\section{Introduction}

52 Glucocorticoids, such as hydrocortisone and dexamethasone, are potent anti-inflammatory agents

53 that are widely used in the treatment of various disorders, such as autoimmune diseases,

54 bronchial asthma, and transplant rejection. However, high-dose and long-term of glucocorticoid

55 treatment may lead to osteoporosis and osteonecrosis (Kerachian et al. 2009) by promoting

56 apoptosis of osteoblasts and mature osteocytes, extending the life span of osteoclasts, as well as

57 suppressing proliferation and terminal differentiation of preosteoblasts(Carcamo-Orive et al.

58 2010). Glucocorticoid-induced osteoporosis occur in about 30-50\% long-term users of

59 glucocorticoids, representing the most common cause of secondary osteoporosis(Hayat \&

60 Magrey 2020; van Staa et al. 2002). Studies have shown that the dose and duration of

61 glucocorticoid treatment determine bone fragility(Barturen et al. 2020) and that high-dose and

62 long-term intake of glucocorticoids is a major contributor to osteoblast and osteocyte

63 apoptosis(Hsu \& Nanes 2017; Weinstein 2011). Thus, the identification of key molecules

64 involved in glucocorticoid-induced osteoporosis is urgently needed for improving bone

65 regeneration in glucocorticoid therapies.

66 The transforming growth factor beta (TGF- $\beta$ )/TGF- $\beta$ receptors (TGFBRs)/Smads signaling

67 pathway activates osteogenesis through type I and type II serine/threonine kinase receptors

68 TGFBR1 and TGFBR2(Nguyen et al. 2013; Wu et al. 2016). TGFBR1 is phosphorylated after

69 the activation of TGFBR2(Wagner et al. 2010), which initiates intracellular signaling through

70 phosphorylation of receptor-regulated Smads (R-Smads). The activated R-Smads form

71 complexes with common-partner Smads (co-Smads) and Smad4 and then translocate into the

72 nucleus to direct transcriptional response(Yi et al. 2010). Studies have shown that TGF-

$73 \beta$ /TGFBRs/Smads signaling is inhibited in osteoporosis and osteonecrosis of the femoral head 
74 induced by glucocorticoids ( $\mathrm{Li}$ et al. 2017; Tao et al. 2017; Xie et al. 2018); however, the

75 underlying mechanism remains unclear.

76 Core fucosylation catalyzed by $\alpha-1,6$ fucosyltransferase 8 (FUT8) is the transfer of fucose from

77 GDP-fucose to the core structure of the N-glycan chain (Angie D. Calderon 2017). Abnormal

78 core fucosylation contributes to multiple pathological conditions, such as cancer, pulmonary

79 emphysema, and tissue remodeling(Bastian et al. 2021; Iijima et al. 2017; Wang et al. 2017).

80 However, the association of core fucosylation with bone formation remains unclear. It has been

81 reported that FUT8-mediated core fucosylation is crucial for TGF- $\beta$ binding to TGFBRs. FUT8

82 deficiency-induced deficit of core fucosylation results in impaired binding of TGF- $\beta$ to TGFBRs,

83 decreased TGFBR activation, as well as inhibited downstream signaling(Schachter 2005;

84 Venkatachalam \& Weinberg 2013). Our previous study has shown that fucosylation is

85 downregulated in glucocorticoid-induced osteonecrosis of the femoral head(Song et al. 2018),

86 suggesting that FUT8-mediated core fucosylation may play a role in osteogenesis. Therefore, we

87 hypothesized that glucocorticoids, such as dexamethasone, might inhibit osteogenic

88 differentiation of preosteoblasts by impairing the core fucosylation and that overexpression of

89 FUT8 might reverse the inhibitory effect of dexamethasone on osteogenic differentiation by

90 activating TGF- $\beta$ /TGFBRs/Smads signaling.

91 Mouse calvaria-derived osteoblast precursor cell line MC3T3-E1 possesses the majority of the

92 molecular features of osteocytes(Bhalerao et al. 1995) and has been widely used to study the

93 effect of TGF- $\beta$ signaling on osteogenesis(Chen et al. 2019; Li \& Jiang 2019; Wang et al. 2012).

94 In this study, we investigated the role and the underlying mechanism of FUT8 in the regulation

95 of osteogenic response in MC3T3-E1 cells exposed to high-dose dexamethasone. Our findings

96 suggest that overexpression of FUT8 may counter the inhibitory effect of high-dose 
97 dexamethasone on osteogenic response of preosteoblasts, serving as a potential therapeutic

98 strategy for promoting bone formation in glucocorticoid therapy.

99

100 Materials \& Methods

101 Cell culture and dexamethasone treatment

102 Murine osteoblast precursor cell line MC3T3-E1 was obtained from Shanghai Zhong Qiao Xin

103 Zhou Biotechnology (Shanghai, China). Cells were maintained in $\alpha$-MEM (Gibco, Gaithersburg,

104 MD, USA) supplemented with 10\% fetal bovine serum (FBS), $50 \mu \mathrm{M}$ ascorbate, $1 \%$ penicillin-

105 streptomycin, and $10 \mathrm{mM} \beta$-glycerophosphate in a humidified atmosphere with $5 \% \mathrm{CO}_{2}$ at $37^{\circ} \mathrm{C}$.

106 Cells at the third or fourth passage were used in the experiments. For dexamethasone treatment,

107 cells were cultured in $\alpha$-MEM supplemented with $1 \%$ FBS for $24 \mathrm{~h}$ and then stimulated with

108 different concentrations of dexamethasone $\left(0,10^{-8}, 10^{-7}, 10^{-6}\right.$, and $10^{-5} \mathrm{M}$; Macklin, Shanghai,

109 China)(Belka et al. 2019).

110 Plasmid construction and transfection

111 Mouse FUT8 was amplified using primers 5'-

112 TACAAGTCCGGACTCAGATCTGCCACCATGCGGGCATGGACTGGT-3' (sense) and 5'-

113 GTACCGTCGACTGCAGAATTCCTATTTTTCAGCTTCAGGATATGTGG-3' (antisense) and

114 cloned into pEGFP-C1 vector (Clontech, Mountain View, CA, USA). Mouse TGF- $\beta 1$ was

115 amplified using primers 5'-

116 CACTAGTCCAGTGTGGGAATTCGCCACCATGCCGCCCTCGGGGCTG (sense)-3' and 5'-

117 GCCCTCTAGACTCGAGCGGCCGCTCAGCTGCACTTGCAGGAGC-3' (antisense) and

118 cloned into pcDNA3.1-V5-HisB vector (V81020; Invitrogen, Carlsbad, CA, USA). The clones

119 were confirmed by DNA sequencing. MC3T3-E1 cells were transiently transfected with 
120 corresponding vectors using Lipofectamine ${ }^{\circledR} 3000$ (Invitrogen).

\section{Cell proliferation assay}

122 Cell proliferation was examined using the cell counting kit-8 (CCK8) assay. Briefly, MC3T3-E1

123 cells were seeded in a 96-well plate at a density of 1,000 cells/well and cultured for $24 \mathrm{~h}$ at $37^{\circ} \mathrm{C}$.

124 Cells were treated with different concentrations of dexamethasone as above mentioned.

125 Phosphate buffered saline (PBS) was used as a vehicle. At 24 h, 48 h, 72 h, 7 d, or 14 d after

126 treatment, cells were incubated with CCK8 solution $(10 \mu \mathrm{L} /$ well $)$ for $1 \mathrm{~h}$ at $37^{\circ} \mathrm{C}$. The

127 absorbance was measured at $450 \mathrm{~nm}$ wavelength using a Labserv K3 microplate reader (Thermo

128 Fisher Scientific, Waltham, MA, USA).

\section{Alkaline phosphatase staining}

130 MC3T3-E1 cells were cultured in osteogenic medium containing $50 \mu \mathrm{M}$ ascorbate and $10 \mathrm{mM} \beta-$

131 glycerophosphate for 7 or 14 days, washed with ice-cold PBS, and fixed with 95\% ice-cold

132 ethanol for 30 min. Alkaline phosphatase (ALP) staining was performed using an ALP staining

133 kit (Beyotime, Guangzhou, China) following the manufacturer's instruction. ALP staining was

134 observed using a 450-fluorescent inverted phase-contrast microscope (Olympus, Tokyo, Japan)

\section{Alizarin red staining}

136 Alizarin red staining was performed to examine mineralization of MC3T3-E1 cells. Cells were

137 cultured in osteogenic medium for 7 or 14 days and then fixed in 4\% paraformaldehyde (Sigma-

138 Aldrich, St. Louise, MO, USA) for 30 min. After 3 washes with ice-cold PBS, cells were stained

139 with Alizarin red (Sigma-Aldrich) for 5 min. Alizarin red staining was observed using an

140 Olympus 450-fluorescent inverted phase-contrast microscope.

\section{Quantitative real-time PCR (qRT-PCR)}

142 Total RNA was isolated from MC3T3-E1 cells using Trizol reagent (Invitrogen). qRT-PCR was 
143 performed using a SYBR Green PCR kit (Invitrogen) and gene-specific primers (Table 1).

144 ACTB was used as an internal reference. The relative gene expression was determined using the $1452^{-\Delta \Delta \mathrm{Ct}}$ method. The PCR reactions were performed in triplicates.

\section{Western blot analysis}

147 Proteins were isolated from MC3T3-E1 cells using RIPA buffer. A total of $60 \mu \mathrm{g}$ protein 148 samples were separated using $10 \%$ SDS-PAGE and transferred to a polyvinylidene difluoride

149 (PVDF) membrane. The membrane was incubated with primary antibody against Smad2/Smad3

150 (1:1,000; 5678S, Cell Signaling Technology), p-Smad2(Ser465/467)/Smad3(Ser423/425)

151 (1:1,000; 8828S, Cell Signaling Technology), and $\beta$-actin (1:1,000; 4970T, Cell Signaling

152 Technology) Incubated overnight at $4^{\circ} \mathrm{C}$. The membrane was washed 3 times with TBST and

153 incubated with HRP-linked secondary antibody (1:1,000; 7074P2; Cell Signaling Technology)

154 for 1 hour at room temperature.

\section{Lectin blot analysis}

156 Lens Culinaris Agglutinin (LCA) lectin blot analysis was performed to detect the $\alpha 1,6$

157 fucosylated trimannose-core structure of N-linked oligosaccharide(Imai-Nishiya et al. 2007).

158 Proteins were isolated from cells using RIPA buffer. A total of $60 \mu \mathrm{g}$ proteins per sample were

159 separated by $10 \%$ SDS-PAGE and transferred to a PVDF membrane. The membrane was

160 blocked using 5\% fat-free milk in TBST at room temperature overnight, followed by 3 washes

161 with TBST. Then, the membrane was incubated with $3 \mu \mathrm{g} / \mathrm{mL}$ biotinylated-LCA (1:1000; B-

162 1045; Maravai Life Sciences, San Diego, CA, USA) in 5\% fat-free milk overnight at $4^{\circ} \mathrm{C}$. After

1633 washes with TBST, the membrane was incubated with HRP-conjugated streptavidin (1:1,000;

164 E030100-01; EathOx life science, Millbrae, CA, USA) for $1 \mathrm{~h}$ at room temperature. After an

165 incubation with Western blot substrate solution for $2 \mathrm{~min}$, the images were acquired in a 
166 darkroom. Data was analyzed using ImageJ software (National Institutes of Health, Bethesda, 167 MD, USA).

168 Co-immunoprecipitation (Co-IP)

169 Co-IP was performed using a Beaver Beads protein A/G immunoprecipitation kit (22202-20;

170 Beaver Nano-Technologies, China) following the manufacturer's instruction. Briefly, cell lysates

171 were centrifuged at $12,000 \mathrm{rpm}$ for $20 \mathrm{~min}$ at $4^{\circ} \mathrm{C}$. The supernatant was collected, and $500 \mu \mathrm{g}$

172 proteins were incubated with $2 \mu \mathrm{g}$ anti-TGFBR1 (ab235178; Abcam, Cambridge, UK) or anti-

173 TGFBR2 antibody (ab269279; Abcam)-conjugated protein A/G agarose beads overnight at $4{ }^{\circ} \mathrm{C}$.

174 The immunoprecipitates were collected and washed three times with lysis buffer. Equal amounts

$175(10 \mu \mathrm{g} /$ lane $)$ of proteins were subjected to 12\% SDS-PAGE for LCA blot analysis.

176 Immunofluorescence staining

177 MC3T3-E1 cells were seeded in a 6-well plate at a density of $2 \times 10^{5}$ cells $/$ well. After treatment,

178 cells were washed 3 times with PBS, followed by fixation with 4\% paraformaldehyde for 15

179 min. Cells were then treated with $0.1 \%$ PBS-Triton X-100 for 5 min and incubated with

180 rhodamine-conjugated LCA (1:1000; RL-1042-5; Vector Laboratories, Burlingame, CA, USA)

181 for $1 \mathrm{~h}$ at $26-28^{\circ} \mathrm{C}$. The nucleus was stained with DAPI (S2110; Solarbio) for $1 \mathrm{~min}$. The

182 fluorescent staining was observed using an Olympus CKX53 inverted microscope. Images were

183 acquired using an UPlanFLN objective at magnification $40 \times$ and analyzed using VistarImage 3.0

184 software (Nikon, Japan).

185 Indirect TGF-ß1 binding assay

186 The pcDNA3.1-V5-HisB vector overexpressing His-TGF- $\beta 1$ was constructed as above

187 mentioned and transfected into MC3T3-E1 cells. The conditioned medium containing His-TGF-

$188 \beta 1$ was collected at $48 \mathrm{~h}$ after transfection. MC3T3-E1 cells transfected with FUT8-

Peer] reviewing PDF | (2021:06:63083:1:0:NEW 19 Sep 2021) 
189 overexpressing vectors or empty vectors were incubated with the conditioned medium for $4 \mathrm{~h}$ at

$1904^{\circ} \mathrm{C}$, followed by cell lysis using the lysis buffer containing $25 \mathrm{mM}$ Hepes, $150 \mathrm{mM} \mathrm{NaCl}, 5 \mathrm{mM}$

191 EDTA, $10 \mu \mathrm{g} / \mathrm{ml}$ aprotinin, $5 \mu \mathrm{g} / \mathrm{ml}$ leupeptin, $10 \%$ glycerol, and 1\% Triton X-100 (pH 7.6). Cell

192 lysates were collected by centrifugation at $10,000 \times g$ for $15 \mathrm{~min}$. The binding of His-TGF- $\beta 1$

193 and endogenous TGFBRs in MC3T3-E1 cells was detected using an ALP-conjugated anti-His

194 antibody (ab49746, Abcam). The bound ALP was measured using a p-nitrophenyl phosphate

195 substrate (Sigma-Aldrich) to quantify the binding of His-TGF- $\beta 1$ and TGFBRs(Tu et al. 2017).

196 Statistical analysis

197 Data are expressed as the mean \pm standard deviation. Statistical analysis was performed using

198 SPSS 23.0 software (SPSS Inc., Chicago, IL, USA). Comparisons among different groups were

199 performed using one-way analysis of variance followed by Tukey's post-hoc test. A $P$ value less

200 than 0.05 was considered statistically significant.

201

202

Results

203

High-dose dexamethasone inhibits cell proliferation, osteogenic differentiation, and FUT8

204

mRNA expression of MC3T3-E1 cells

205 To investigate the effect of dexamethasone on preosteoblast proliferation, we treated MC3T3-E1

206 cells with different concentrations of dexamethasone and examined the cell viability at different

207 time points. The results of CCK8 assay showed that compared with vehicle treatment, low-dose

208 dexamethasone $\left(10^{-8}\right.$ and $\left.10^{-7} \mathrm{M}\right)$ significantly promoted cell proliferation in a time-dependent

209 manner, whereas high-dose dexamethasone $\left(10^{-6}\right.$ and $\left.10^{-5} \mathrm{M}\right)$ significantly inhibited cell

210 proliferation with maximum effects at 7 days after treatment in MC3T3-E1 cells (Figure 1A).

211 Then, we examined the effects of dexamethasone on osteoblast differentiation and mineralization 
212 of MC3T3-E1 cells. The results of ALP staining (Figure 1B, upper panel) and Alizarin red

213 staining (Figure 1B, lower panel) showed that compared with control cells, cells treated with

214 low-dose dexamethasone exhibited stronger ALP and Alizarin red staining, whereas those treated

215 with high-dose dexamethasone demonstrated weaker staining (Figure 1B). These data suggest

216 that low-dose dexamethasone promotes, whereas high-dose dexamethasone inhibits cell

217 proliferation, osteoblast differentiation, and mineralization of preosteoblasts.

218 To further examine the effect of dexamethasone treatment on osteogenesis and the involvement

219 of core fucosylation, we determined the mRNA levels of FUT8 as well as osteogenesis- and

220 adipogenesis-related genes in MC3T3-E1 cells exposed to different doses of dexamethasone. To

221 avoid the substantial changes in cell numbers resulting from long-term exposure to

222 dexamethasone (Figure 1A), we treated the cells for 3 day. The results showed that low-dose

223 dexamethasone significantly upregulated mRNA expression of FUT8 whereas high-dose

224 dexamethasone dramatically downregulated mRNA expression of FUT8 (Figure 1C, upper left

225 panel). Low-dose dexamethasone also significantly upregulated mRNA expression of

226 osteogenesis-related genes, including ALP, BMP2, Osx, Ocn, and Runx2, whereas high-dose

227 dexamethasone generally showed no effects on mRNA expression of these gens (Figure 1C,

228 upper and middle panels). In contrast, low-dose dexamethasone generally downregulated mRNA

229 expression of adipogenesis-related genes, including CEBP $\alpha, \mathrm{HDAC} 5$, and PPAR $\gamma$, whereas

230 high-dose dexamethasone generally, significantly upregulated these gens (Figure 1C, lower

231 panel). These data suggest that high-dose dexamethasone supports adipogenesis but inhibits

232 osteogenesis, involving the downregulation of FUT8 expression.

233 Overexpression of FUT8 promotes osteogenic differentiation of MC3T3-E1 cells regardless of

234 the presence or absence of high-dose dexamethasone 
235 To examine whether compensatory expression of FUT8 could reverse the effect of high-dose

236 dexamethasone on osteogenesis, we overexpressed FUT8 in MC3T3-E1 cells and then treated

237 the cells with vehicle or high-dose dexamethasone. As shown in Figure 2A, compared with

238 empty vector transfection, overexpression of FUT8 enhanced the intensities of ALP and Alizarin

239 red staining, in the absence of dexamethasone. High-dose dexamethasone treatment resulted in

240 reductions in ALP and Alizarin red staining in empty vector-transfected cells. Importantly,

241 overexpression of FUT8 enhanced ALP and Alizarin red staining in the presence of high-dose

242 dexamethasone, suggesting that overexpression of FUT8 abrogates the inhibitory effects of high-

243 dose dexamethasone on osteogenic differentiation and mineralization of preosteoblasts. Then, we

244 measured the mRNA levels of osteogenic markers in MC3T3-E1 cells in response to FUT8

245 overexpression. As shown in Figure 2B, compared with control, FUT8 overexpression

246 dramatically promoted mRNA expression of osteogenic markers, regardless of the presence or

247 absence of dexamethasone. Collectively, the combined results of Figure 1 and 3 suggest that

248 overexpression of FUT8 reverses the effects of high-dose dexamethasone on osteogenesis.

249 Overexpression of FUT8 activates TGF-ß/Smad signaling in MC3T3-E1 cells in the presence

250 of high-dose dexamethasone

251 Considering the critical role of TGF- $\beta /$ Smad signaling in osteogenesis and the essential role of

252 core fucosylation in TGF- $\beta$ signaling activation, we examined the effect of FUT8 overexpression

253 on TGF- $\beta /$ Smad signaling in MC3T3-E1 preosteoblasts exposed to high-dose dexamethasone.

254 qRT-PCR revealed that in general, overexpression of FUT8 remarkably enhanced mRNA

255 expression of TGF- $\beta 1$, TGF- $\beta 2$, Smad2, Smad3, Smad4, and TGFBR2, while attenuating mRNA

256 expression of TGF- $\beta 3$ and TGFBR1 (Figure 3A-C), regardless of the presence and absence of

257 dexamethasone. Of note, overexpression of FUT8 induced opposite effects between mRNA 
258 expression of TGF- $\beta 1 / 2$ and TGF- $\beta 3$ (Figure $3 \mathrm{~A}$ ) as well as between mRNA expression of

259 TGFBR1 and TGFR2 (Figure 3C). In addition, overexpression of FUT8 did not affect the

260 expression of TGF- $\beta 2$ or Smad4 in the presence of $10^{-6} \mathrm{M}$ dexamethasone or the expression of

261 TGF- $\beta 3$ or TGFBR1 in the presence of $10^{-5} \mathrm{M}$ dexamethasone (Figures 4A and 4C), suggesting

262 that the concentration of dexamethasone may play a role in FUT8-mediated regulation of

263 TGF $\beta /$ Smad signaling.

264 To further investigate whether FUT8 activates TGF- $\beta /$ Smad signaling, we determined the 265 phosphorylation status of Smad2/3 in MC3T3-E1 cells overexpressing FUT8. As shown in 266 Figure 3D, compared with empty vector transfection, overexpression of FUT8 markedly elevated

267 the protein levels of total Smad2/3 but reduced phosphorylated Smad2/3 (p-Smad2/3) in the 268 absence of dexamethasone. On the other hand, treatment with high-dose dexamethasone 269 significantly reduced protein levels of total and phosphorylated Smad2/3, suggesting that high270 dose dexamethasone suppresses Smad signaling in preosteroblasts. Importantly, in the presence

271 of high-dose dexamethasone, overexpression of FUT8 substantially elevated the protein levels of 272 total and $\mathrm{p}-\mathrm{Smad} 2 / 3$, compared with empty vector transfection (Figure 3D). These results

273 suggest that overexpression of FUT8 reverses the inhibitory effect of high-dose dexamethasone 274 on TGF- $\beta / \mathrm{Smad} 2 / 3$ signaling in preostroblasts.

275 Overexpression of FUT8 promotes core fucosylation of TGFBRs and TGF-ß1 binding to 276 TGFBRs in MC3T3-E1 cells in the presence of high-dose dexamethasone

277 Next, we detected $\alpha 1$,6-fucosylated trimannose-core structure in MC3T3-E1 cells to investigated 278 whether FUT8-mediated core fucosylation affects TGF- $\beta 1 /$ TGFBRs signaling of preosteoblasts.

279 LCA immunofluorescent staining showed that overexpression of FUT8 substantially promoted 280 core fucosylation compared with the control, whereas high-dose dexamethasone treatment 
281 significantly suppressed core fucosylation of MC3T3-E1 preosteoblasts. Of note, overexpression

282 of FUT8 partially but significantly counteracted the suppressing effect of high-dose

283 dexamethasone on core fucosylation (Figure 4A). Consistent results were observed in LCA blot

284 analysis (Figure 4B). These data suggest that overexpression of FUT8 reverses the suppressing

285 effect of high-dose dexamethasone on core fucosylation in preosteoblasts.

286 To identify the downstream molecule of FUT8 in preosteoblasts exposed to high-dose

287 dexamethasone, we detected fucosylated-TGFBR1 and TGFBR2 in MC3T3-E1 cells. The results

288 of Co-IP revealed that compared with empty vector transfection, overexpression of FUT8

289 dramatically elevated the TGFBR1-LCA/TGFBR1 ratio but not TGFBR2-LCA/TGFBR2 ratio in

290 MC3T3-E1 cells. Conversely, $10^{-5} \mathrm{M}$ and 10-6 $\mathrm{M}$ dexamethasone significantly reduced TGFBR1-

291 LCA/TGFBR1 ratio and TGFBR2-LCA/TGFBR2 ratio, respectively, in MC3T3-E1 cells,

292 compared with vehicle treatment. Importantly, overexpression of FUT8 significantly reversed the

293 effects of high-dose dexamethasone on the ratios of TGFBR1-LCA/TGFBR1 and TGFBR2-

294 LCA/TGFBR2 (Figure 4C). This finding suggests that overexpression of FUT8 counteracts the

295 inhibitory effect of high-dose dexamethasone on core fucosylation of TGFBR1 and TGFBR2,

296 thereby promoting osteogenesis by activating TGF- $\beta$ signaling in preosteoblasts.

297 To further confirm the enhancive role of FUT8 in TGF- $\beta$ signaling, we examined the change in

298 TGF- $\beta 1$ binding to TGFBRs in response to FUT8 overexpression. As shown in Figure 4D, His-

299 TGF- $\beta 1$ overexpression resulted in a remarkable increase in ALP signal, suggesting that the

300 intensity of ALP signal may reflect the level of His-TGF- $\beta 1$ binding to TGFBRs. Therefore, we 301 showed His-TGF- $\beta 1$ overexpression normalized with the empty vector (His-TGF- $\beta 1$ relative to

302 vector). In the absence of dexamethasone, compared with empty pEGFP-C1 vector transfection, 303 overexpression of FUT8 dramatically elevated the intensity of ALP signal, suggesting that 
304 overexpression of FUT8 promotes His-TGF- $\beta 1$ binding to TGFBRs. In contrast, in empty

305 pEGFP-C1 vector-transfected cells, high-dose dexamethasone significantly reduced the intensity

306 of ALP signal compared with vehicle treatment, suggesting that high-dose dexamethasone

307 inhibits His-TGF- $\beta 1$ binding to TGFBRs. Of note, overexpression of FUT8 dramatically

308 reversed the inhibitory effects of high-dose dexamethasone on His-TGF- $\beta 1$ binding to TGFBRs.

309 Together, these data suggest that FUT8 may promote TGF- $\beta 1$ binding to TGFBRs and then

310 activate downstream signals, facilitating osteogenesis in the presence of high-dose

311 dexamethasone.

312

313 Discussion

314 Glucocorticoids exert dual effects on skeletal metabolism and osteogenesis(Hartmann et al.

315 2016). Glucocorticoids at physiological levels promote osteoblast proliferation and osteogenic

316 differentiation. In contrast, glucocorticoids at pharmacological doses may induce apoptosis of

317 osteoblasts and osteocytes while inhibiting proliferation and differentiation of osteoprogenitor

318 cells(Phillips et al. 2006). Thus, different doses of dexamethasone may exert different effects on

319 osteogenesis. A recent study has found that treatment with dexamethasone $(100 \mathrm{nM})$ for $6 \mathrm{~h}$

320 significantly downregulates FUT8 expression in human corneal epithelial cells(Kadmiel et al.

321 2016). In addition, our previous study has demonstrated that fucosylation is downregulated in the

322 glucocorticoid-induced osteonecrosis of the femoral head(Song et al. 2018). These findings

323 suggest that glucocorticoid treatment is associated with dysregulated fucosylation, possibly

324 playing an important role in glucocorticoid-induced imbalance in bone formation and resorption.

325 To verify the effect of glucocorticoid treatment on FUT8 expression and osteogenic response, we

326 treated MC3T3-E1 preosteoblasts with different doses of dexamethasone. We found that 
327 mRNA expression of FUT8 was significantly downregulated in response to high-dose

328 dexamethasone stimulation, along with significantly suppressed cell proliferation and osteogenic

329 differentiation, suggesting that high-dose dexamethasone suppresses osteogenesis possibly by

330 downregulating FUT8-mediated core fucosylation of osteogenesis-related glycoproteins. The

331 canonical TGF- $\beta$ pathway facilitates bone formation(Baffi et al. 2006; Liang et al. 2020; Peters

332 et al. 2017; Wang et al. 2013). Of note, FUT8-mediated core fucosylation is essential for TGF- $\beta$

333 binding to TGFBRs(Schachter 2005; van Staa et al. 2002), and TGF- $\beta$ /TGFBRs/Smads signaling

334 is inhibited in glucocorticoid-induced osteoporosis and osteonecrosis(Li et al. 2017; Tao et al.

335 2017; Xie et al. 2018). Thus, we hypothesized that high-dose dexamethasone suppresses

336 osteogenesis possibly through downregulating FUT8-mediated core fucosylation of TGF-

$337 \beta$ /TGFBRs and that compensatory expression of FUT8 might reverse the effects of high-dose

338 dexamethasone. Indeed, our results showed that overexpression of FUT8 reversed the inhibitory

339 effects of high-dose dexamethasone on TGF $\beta /$ Smad signaling, core fucosylation of TGFBRs, as

340 well as TGF- $\beta 1$ binding to TGFBRs in MC3T3-E1 preosteoblasts. Together, these events

341 facilitate osteogenic differentiation of preosteoblasts, as evidenced by enhanced ALP and

342 Alizarin red staining and mRNA expression of osteogenic markers in MC3T3-E1 in the presence

343 of high-dose dexamethasone.

344 FUT8-mediated core fucosylation plays an essential role in the activation of TGF- $\beta$ signaling.

345 Dysregulation of FUT8 blocks the activation of TGF $\beta /$ Smad signaling(Ng et al. 2018; Wang et

346 al. 2005). Tu et al. have reported that FUT8-mediated TGFBR core fucosylation promotes TGF-

$347 \beta$ signaling and epithelial-mesenchymal transition, stimulating breast cancer cell invasion and

348 metastasis(Tu et al. 2017). However, whether FUT8 regulates core fucosylation of TGFBRs in

349 preosteoblasts exposed to glucocorticoids remains unexplored. In this study, the results of Co-IP 
350 revealed that overexpression of FUT8 dramatically elevated the TGFBR1-LCA/TGFBR1 ratio in

351 the absence of dexamethasone and reversed the effects of high-dose dexamethasone on the ratios

352 of TGFBR1-LCA/TGFBR1 and TGFBR2-LCA/TGFBR2. This finding suggests that FUT8

353 mediates core fucosylation of TGFBRs in preosteoblasts, thus promoting osteogenesis by

354 activating TGF- $\beta$ signaling.

355

356 Conclusions

357 In conclusion, high-dose dexamethasone attenuated FUT8 mRNA expression in MC3T3-E1

358 preosteoblasts. Overexpression of FUT8 reversed the inhibitory effects of high-dose

359 dexamethasone on osteogenic differentiation, TGF- $\beta /$ Smads signaling, core fucosylation of

360 TGFBRs, as well as TGF- $\beta 1$ binding to TGFBRs in MC3T3-E1 cells. These findings suggest

361 that overexpression of FUT8 is as a potential therapeutic approach to counteract the side effects

362 of glucocorticoid therapy through activating TGF- $\beta$ signaling.

363

Peer] reviewing PDF | (2021:06:63083:1:0:NEW 19 Sep 2021) 


\section{Acknowledgments}

365 We thank Ying Li for her help in the amplification of plasmids and some experimental supplies

366 Funding.

367

368 Funding

369 This work was supported by the National Natural Science Foundation of China [grant numer 37081904226 to Ziqi Li].

371

372 Data Sharing Statement

373 The datasets used and/or analysed during the current study are available from the corresponding

374 author on reasonable request.

375

376 Conflicts of Interest

377 The authors have no conflicts of interest to declare.

378

\section{Author Contributions}

380 Zhiming $\mathrm{Wu}$ is the main author of the article, completes the collection and analysis of relevant

381 literature data and the writing of the first draft of the paper; Tianye Lin and Pan Kang participate 382 in the analysis and sorting of literature data; Ziqi Li is the creator and person in charge of the 383 project and guides the designing of thesis. Zhikun Zhuang, Haibin Wang, Wei He, Qiushi Wei 384 critically revised the manuscript. All authors have read and critically revised the manuscript. 385 Zhiming Wu, Tianye Lin and Pan Kang contributed equally to this work. 
387

388

389

390

391

392

393

394

395

396

397

398

399

400

401

402

403

404

405

406

407

408

409

\section{References}

Angie D. Calderon LLaPGW. 2017. Angie D. Calderon, Lei Li and Peng G. Wang. Pure and Applied Chemistry 89.

Baffi MO, Moran MA, and Serra R. 2006. Tgfbr2 regulates the maintenance of boundaries in the axial skeleton. Developmental Biology 296:363-374. 10.1016/j.ydbio.2006.06.002

Barturen G, Babaei S, Catala-Moll F, Martinez-Bueno M, Makowska Z, Martorell-Marugan J, Carmona-Saez P, Toro-Dominguez D, Carnero-Montoro E, Teruel M, Kerick M, AcostaHerrera M, Le Lann L, Jamin C, Rodriguez-Ubreva J, Garcia-Gomez A, Kageyama J, Buttgereit A, Hayat S, Mueller J, Lesche R, Hernandez-Fuentes M, Juarez M, Rowley T, White I, Maranon C, Gomes Anjos T, Varela N, Aguilar-Quesada R, Garrancho FJ, Lopez-Berrio A, Rodriguez Maresca M, Navarro-Linares H, Almeida I, Azevedo N, Brandao M, Campar A, Faria R, Farinha F, Marinho A, Neves E, Tavares A, Vasconcelos C, Trombetta E, Montanelli G, Vigone B, Alvarez-Errico D, Li T, Blanco Alonso R, Corrales Martinez A, Genre F, Lopez Mejias R, Gonzalez-Gay MA, Remuzgo S, Ubilla Garcia B, Cervera R, Espinosa G, Rodriguez-Pinto I, De Langhe E, Cremer J, Lories R, Belz D, Hunzelmann N, Baerlecken N, Kniesch K, Witte T, Lehner M, Stummvoll G, Zauner M, Aguirre-Zamorano MA, Barbarroja N, Castro-Villegas MC, Collantes-Estevez E, de Ramon E, Diaz Quintero I, Escudero-Contreras A, Fernandez Roldan MC, Jimenez Gomez Y, Jimenez Moleon I, Lopez-Pedrera R, Ortega-Castro R, Ortego N, Raya E, Artusi C, Gerosa M, Luigi Meroni P, Schioppo T, De Groof A, Ducreux J, Lauwerys B, Maudoux AL, Cornec D, Devauchelle-Pensec V, Jousse-Joulin S, Jouve PE, Rouviere B, Saraux A, Simon Q, Alvarez M, Chizzolini C, Dufour A, Wynar D, Balog A, Bocskai M, Deak M, Dulic S, Kadar G, Kovacs L, Cheng Q, Gerl V, Hiepe F, Khodadadi L, Thiel S, 
410

411

412

413

414

415

416

417

418

419

420

421

422

423

424

425

426

427

428

429

430

431

432

de Rinaldis E, Rao S, Benschop RJ, Chamberlain C, Dow ER, Ioannou Y, Laigle L, Marovac J, Wojcik J, Renaudineau Y, Borghi MO, Frostegard J, Martin J, Beretta L, Ballestar E, McDonald F, Pers JO, and Alarcon-Riquelme ME. 2020. Integrative Analysis Reveals a Molecular Stratification of Systemic Autoimmune Diseases. Arthritis Rheumatol. 10.1002/art.41610

Bastian K, Scott E, Elliott DJ, and Munkley J. 2021. FUT8 Alpha-(1,6)-Fucosyltransferase in Cancer. International Journal of Molecular Sciences 22. 10.3390/ijms22010455

Belka J, Nickel J, and Kurth DG. 2019. Growth on Metallo-Supramolecular Coordination Polyelectrolyte (MEPE) Stimulates Osteogenic Differentiation of Human Osteosarcoma Cells (MG63) and Human Bone Marrow Derived Mesenchymal Stem Cells. Polymers (Basel) 11. 10.3390/polym11071090

Bhalerao J, Bogers J, Van Marck E, and Merregaert J. 1995. Establishment and characterization of two clonal cell lines derived from murine mandibular condyles. Tissue and Cell 27:369-382. 10.1016/s0040-8166(95)80058-1

Carcamo-Orive I, Gaztelumendi A, Delgado J, Tejados N, Dorronsoro A, Fernandez-Rueda J, Pennington DJ, and Trigueros C. 2010. Regulation of human bone marrow stromal cell proliferation and differentiation capacity by glucocorticoid receptor and AP-1 crosstalk. Journal of Bone and Mineral Research 25:2115-2125. 10.1002/jbmr.120

Chen Y, Sun C, Lu J, Zou L, Hu M, Yang Z, and Xu Y. 2019. MicroRNA-590-5p antagonizes the inhibitory effect of high glucose on osteoblast differentiation by suppressing Smad7 in MC3T3-E1 cells. Journal of International Medical Research 47:1740-1748. $10.1177 / 0300060519830212$

Hartmann K, Koenen M, Schauer S, Wittig-Blaich S, Ahmad M, Baschant U, and Tuckermann 
433

434

435

436

437

438

439

440

441

442

443

444

445

446

447

448

449

450

451

452

453

454

455

JP. 2016. Molecular Actions of Glucocorticoids in Cartilage and Bone During Health, Disease, and Steroid Therapy. Physiological Reviews 96:409-447.

10.1152/physrev.00011.2015

Hayat S, and Magrey MN. 2020. Glucocorticoid-induced osteoporosis: Insights for the clinician. Cleveland Clinic Journal of Medicine 87:417-426. 10.3949/ccjm.87a.19039

Hsu E, and Nanes M. 2017. Advances in treatment of glucocorticoid-induced osteoporosis. Current Opinion in Endocrinology, Diabetes, and Obesity 24:411-417. 10.1097/MED.0000000000000368

Iijima J, Kobayashi S, Kitazume S, Kizuka Y, Fujinawa R, Korekane H, Shibata T, Saitoh SI, Akashi-Takamura S, Miyake K, Miyoshi E, and Taniguchi N. 2017. Core fucose is critical for CD14-dependent Toll-like receptor 4 signaling. Glycobiology 27:1006-1015. 10.1093/glycob/cwx075

Imai-Nishiya H, Mori K, Inoue M, Wakitani M, Iida S, Shitara K, and Satoh M. 2007. Double knockdown of alpha1,6-fucosyltransferase (FUT8) and GDP-mannose 4,6-dehydratase (GMD) in antibody-producing cells: a new strategy for generating fully non-fucosylated therapeutic antibodies with enhanced ADCC. BMC Biotechnology 7:84. 10.1186/1472$6750-7-84$

Kadmiel M, Janoshazi A, Xu X, and Cidlowski JA. 2016. Glucocorticoid action in human corneal epithelial cells establishes roles for corticosteroids in wound healing and barrier function of the eye. Experimental Eye Research 152:10-33. 10.1016/j.exer.2016.08.020

Kerachian MA, Seguin C, and Harvey EJ. 2009. Glucocorticoids in osteonecrosis of the femoral head: a new understanding of the mechanisms of action. Journal of Steroid Biochemistry and Molecular Biology 114:121-128. 10.1016/j.jsbmb.2009.02.007 
456 Li L, and Jiang D. 2019. Hypoxia-responsive miRNA-21-5p inhibits Runx2 suppression by

457

458

459

460

461

462

463

464

465

466

467

468

469

470

471

472

473

474

475

476

477

478 targeting SMAD7 in MC3T3-E1 cells. Journal of Cellular Biochemistry 120:1686716875. $10.1002 /$ jcb. 28944

Li Y, Jie L, Tian AY, Zhong S, Tian MY, Zhong Y, Wang Y, Li H, Li J, Sun X, and Du H. 2017. Transforming Growth Factor Beta is regulated by a Glucocorticoid-Dependent Mechanism in Denervation Mouse Bone. Scientific Reports 7:9925. 10.1038/s41598-017$09793-y$

Liang Y, Zhu B, Li S, Zhai Y, Yang Y, Bai Z, Zeng Y, and Li D. 2020. Curcumin protects bone biomechanical properties and microarchitecture in type 2 diabetic rats with osteoporosis via the TGFbeta/Smad2/3 pathway. Experimental and Therapeutic Medicine 20:22002208. 10.3892/etm.2020.8943

Ng BG, Xu G, Chandy N, Steyermark J, Shinde DN, Radtke K, Raymond K, Lebrilla CB, AlAsmari A, Suchy SF, Powis Z, Faqeih EA, Berry SA, Kronn DF, and Freeze HH. 2018. Biallelic Mutations in FUT8 Cause a Congenital Disorder of Glycosylation with Defective Fucosylation. American Journal of Human Genetics 102:188-195. 10.1016/j.ajhg.2017.12.009

Nguyen J, Tang SY, Nguyen D, and Alliston T. 2013. Load regulates bone formation and Sclerostin expression through a TGFbeta-dependent mechanism. PloS One 8:e53813. 10.1371/journal.pone.0053813

Peters SB, Wang Y, and Serra R. 2017. Tgfbr2 is required in osterix expressing cells for postnatal skeletal development. Bone 97:54-64. 10.1016/j.bone.2016.12.017

Phillips JE, Gersbach CA, Wojtowicz AM, and Garcia AJ. 2006. Glucocorticoid-induced osteogenesis is negatively regulated by Runx $2 / \mathrm{Cbfa} 1$ serine phosphorylation. Journal of 
479

480

481

482

483

484

485

486

487

488

489

490

491

492

493

494

495

496

497

498

499

500

501

Cell Science 119:581-591. 10.1242/jcs.02758

Schachter H. 2005. The search for glycan function: fucosylation of the TGF-beta1 receptor is required for receptor activation. Proceedings of the National Academy of Sciences of the United States of America 102:15721-15722. 10.1073/pnas.0507659102

Song T, Chen P, Stroble C, Ruhaak LR, Wang H, Li Z, He W, and Lebrilla CB. 2018. Serum glycosylation characterization of osteonecrosis of the femoral head by mass spectrometry. Eur J Mass Spectrom (Chichester) 24:178-187. $10.1177 / 1469066717740010$

Tao J, Dong B, Yang LX, Xu KQ, Ma S, and Lu J. 2017. TGFbeta1 expression in adults with nontraumatic osteonecrosis of the femoral head. Mol Med Rep 16:9539-9544.

$$
10.3892 / \mathrm{mmr} .2017 .7817
$$

Tu CF, Wu MY, Lin YC, Kannagi R, and Yang RB. 2017. FUT8 promotes breast cancer cell invasiveness by remodeling TGF-beta receptor core fucosylation. Breast Cancer Research 19:111. 10.1186/s13058-017-0904-8

van Staa TP, Leufkens HG, and Cooper C. 2002. The epidemiology of corticosteroid-induced osteoporosis: a meta-analysis. Osteoporosis International 13:777-787.

$$
10.1007 / \mathrm{s} 001980200108
$$

Venkatachalam MA, and Weinberg JM. 2013. New wrinkles in old receptors: core fucosylation is yet another target to inhibit TGF-beta signaling. Kidney International 84:11-14. $10.1038 / \mathrm{ki} .2013 .95$

Wagner DO, Sieber C, Bhushan R, Borgermann JH, Graf D, and Knaus P. 2010. BMPs: from bone to body morphogenetic proteins. Sci Signal 3:mr1. 10.1126/scisignal.3107mr1

Wang N, Deng Y, Liu A, Shen N, Wang W, Du X, Tang Q, Li S, Odeh Z, Wu T, and Lin H. 
502

503

504

505

506

507

508

509

510

511

512

513

514

515

516

517

518

519

520

$521 \mathrm{Wu}$ M, Chen G, and Li YP. 2016. TGF-beta and BMP signaling in osteoblast, skeletal

522

523

524 Xie Y, Hu JZ, and Shi ZY. 2018. MiR-181d promotes steroid-induced osteonecrosis of the 
525 femoral head by targeting SMAD3 to inhibit osteogenic differentiation of hBMSCs.

526 European Review for Medical and Pharmacological Sciences 22:4053-4062.

527 10.26355/eurrev_201807_15393

528 Yi JJ, Barnes AP, Hand R, Polleux F, and Ehlers MD. 2010. TGF-beta signaling specifies axons

529 during brain development. Cell 142:144-157. 10.1016/j.cell.2010.06.010

530 
531 Table 1. Primers for quantitative real-time PCR

\begin{tabular}{|c|c|c|}
\hline Gene & Forward (5'-3') & Reverse (5'-3') \\
\hline ACTB & GAGGTATCCTGACCCTGAAGTA & CACACGCAGCTCATTGTAGA \\
\hline FUT8 & GTGGATGGGAGACTGTGTTTAG & GAGCTCGACCACTTGAATGT \\
\hline ALP & CTTTCGTAGCAGCAGCAAAC & GGAGCGCGTCTTGGATATT \\
\hline $\mathrm{OCN}$ & GGAGCTGCTTTGGTGAGATTAG & GAGTAGCCCAGACTACGGATATT \\
\hline OSX & TGGAGAGGGAAAGGGATTCT & GAAATCTACGAGCAAGGTCTCC \\
\hline RUNX2 & TGGCTTGGGTTTCAGGTTAG & GGTTTCTTAGGGTCTTGGAGTG \\
\hline BMP2 & ACACAGCTGGTCACAGATAAG & CTTCCGCTGTTTGTGTTTGG \\
\hline HDAC5 & AGTACCACACCCTGCTCTAT & AGCATGGCGTACATCTTCTG \\
\hline PPAR $\gamma$ & CTGGCCTCCCTGATGAATAAAG & AGGCTCCATAAAGTCACCAAAG \\
\hline $\mathrm{CEBP} \alpha$ & CTCCCAGAGGACCAATGAAATG & TTAGCCGGAGGAAGCTAAGA \\
\hline TGF $\beta 1$ & GGTGGTATACTGAGACACCTTG & CCCAAGGAAAGGTAGGTGATAG \\
\hline TGF $\beta 2$ & GGCTTTCATTTGGCTTGAGATG & CTTCGGGTGAGACCACAAATAG \\
\hline TGF $\beta 3$ & CGCTACATAGGTGGCAAGAA & CAAGTTGGACTCTCTCСТCAАC \\
\hline Smad2 & GCTGAGTGCCTAAGTGATAGTG & TACAGCCTGGTGGGATCTTA \\
\hline Smad3 & CGCTGTTCCAGTGTGTCTTA & GATGGAGTTCTCTTCCAAGGTC \\
\hline Smad4 & CTGTCTTCATCCGGTCTTCATC & GTTGTTCCTGCTCCACTCAT \\
\hline $\mathrm{T} \beta \mathrm{RI}$ & CCTTGAGTCACTGGGTGTTATG & CCACTTAGCTGTCACCCTAATC \\
\hline $\mathrm{T} \beta \mathrm{RII}$ & GTTCGTGAGCATGGAGAGATAG & CAGGGCTGAGATGATAAGAGTG \\
\hline
\end{tabular}

532

533 


\section{Figure Legends}

535 Figure 1. The effects of dexamethasone on cell proliferation and osteogenic response of

536 MC3T3-E1 cells. (A) MC3T3-E1 cells were cultured in osteogenic medium containing different

537 doses of dexamethasone $\left(0,10^{-8}, 10^{-7}, 10^{-6}\right.$, and $\left.10^{-5} \mathrm{M}\right)$. Cell counting kit-8 assay was performed

538 to measure cell viability at $1 \mathrm{~d}, 2 \mathrm{~d}, 3 \mathrm{~d}, 7 \mathrm{~d}$, and $14 \mathrm{~d}$ after treatment. (B) MC3T3-E1 cells were

539 cultured in osteogenic medium containing different doses of dexamethasone as indicated.

540 Alkaline phosphatase (ALP) staining and Alizarin red staining were conducted to examine

541 osteogenesis and mineralization, respectively. C1: control cells cultured in osteogenic medium

542 without dexamethasone; C2: Control cells cultured in medium without osteogenic supplements.

543 (C) MC3T3-E1 cells were cultured in osteogenic medium containing different doses of

544 dexamethasone as indicated. Quantitative real-time PCR (qRT-PCR) was performed at $3 \mathrm{~d}$ after

545 treatment to measure mRNA expression of FUT8 as well as osteogenesis- and adipogenesis-

546 related genes. ACTB was used as an internal reference. Data are expressed as the mean \pm

547 standard deviation (SD). $* P<0.05, * * P<0.01, * * * P<0.001$ vs. 0 M. All experiments were

548 independently repeated at least 3 times. Dex, Dexamethasone; FUT8, fucosyltransferase 8; ALP:

549 alkaline phosphatase.

550 Figure 2. Overexpression of FUT8 promoted osteogenic differentiation of MC3T3-E1 cells

551 regardless of the presence or absence of high-dose dexamethasone. MC3T3-E1 cells were

552 transiently transfected with empty or FUT8-overexpressing vectors and incubated in osteogenic

553 medium in the absence or presence of high-dose dexamethasone for 5 or 14 days (A) ALP

554 staining and Alizarin red staining were performed to examine osteogenesis and mineralization,

555 respectively (B) qRT-PCR was performed at 3 days after treatment to measure mRNA

556 expression of osteogenesis-related genes. ACTB was used as an internal reference. Data are 
557 expressed as the mean \pm SD. $* P<0.05$, ** $P<0.01$, $* * * P<0.001$, **** $P<0.0001$ vs. pEGFP-

558 C1. All experiments were independently repeated at least 3 times. FUT8, fucosyltransferase;

559 ALP, alkaline phosphatase; Dex, dexamethasone.

560 Figure 3. The effects of overexpression of FUT8 on TGF//Smad signaling in MC3T3-E1

561 cells. MC3T3-E1 cells were transfected with empty or FUT8-overexpressing vectors and

562 cultured in osteogenic medium containing different doses of dexamethasone $\left(0,10^{-6}\right.$, and $\left.10^{-5} \mathrm{M}\right)$

563 for 3 days. (A-C) qRT-PCR was performed to measure mRNA expression of the components of

564 the TGF $\beta /$ Smad pathway. ACTB was used as an internal reference. Data are expressed as the

565 mean $\pm \mathrm{SD} . * P<0.05, * * P<0.01, * * * P<0.001, * * * * P<0.0001$ vs. pEGFP-C1. (D) Western

566 blot analysis was conducted to measure protein expression of phosphorylated and total Smad2/3

567 protein levels. $\beta$-actin was used as an internal reference. All experiments were independently

568 repeated at least 3 times. FUT8, fucosyltransferase; Dex, dexamethasone.

\section{Figure 4. Overexpression of FUT8 promoted core fucosylation of TGFBRs and TGF- $\beta 1$}

570 binding to TGFBRs in MC3T3-E1 cells in the presence of high-dose dexamethasone.

571 MC3T3-E1 cells were transfected with empty or FUT8-overexpressing vectors and cultured in

572 osteogenic medium containing different doses of dexamethasone $\left(0,10^{-6}\right.$, and $\left.10^{-5} \mathrm{M}\right)$ as

573 indicated for 3 days. (A) Lens culinaris agglutinin (LCA)-based immunofluorescence staining

574 was performed to detect $\alpha 1,6$-fucosylated trimannose-core structure in MC3T3-E1 cells. Scale

575 bar $=100 \mu \mathrm{m}$. (B) LCA blot analysis was performed to determine the level of core fucosylated-

576 proteins. (C) Co-immunoprecipitation was conducted to measure the levels of core fucosylated-

577 TGFBR1 and TGFBR2. (D) MC3T3-E1 cells (from A) were transfected with empty or His-

578 tagged TGF- $\beta 1$ (His-TGF- $\beta 1$ )-overexpressing vectors. The conditioned medium was collected at

5792 days after transfection. MC3T3-E1 cells were cultured in the conditioned medium for $4 \mathrm{~h}$, and 
580 the cell lysates were collected. The His-TGF- $\beta 1$ protein bound to endogenous TGFBRs were

581 detected by the ALP-conjugated anti-His antibody and quantified by measuring the bound ALP

582 using a p-nitrophenyl phosphate substrate. Data are expressed as the mean $\pm \mathrm{SD} . * P<0.05, * * P$

$583<0.01, * * * P<0.001, * * * * P<0.0001$ vs. empty vector. mOD405, milli-absorbance units at 405

$584 \mathrm{~nm}$. All experiments were independently repeated at least 3 times. 


\section{Figure 1}

Figure 1

Figure 1. The effects of dexamethasone on cell proliferation and osteogenic response of MC3T3-E1 cells . (A) MC3T3-E1 cells were cultured in osteogenic medium containing different doses of dexamethasone $\left(0,10^{-8}, 10^{-7}, 10^{-6}\right.$, and $\left.10^{-5} \mathrm{M}\right)$. Cell counting kit-8 assay was performed to measure cell viability at $1 \mathrm{~d}, 2 \mathrm{~d}, 3 \mathrm{~d}, 7 \mathrm{~d}$, and $14 \mathrm{~d}$ after treatment. (B) MC3T3-E1 cells were cultured in osteogenic medium containing different doses of dexamethasone as indicated. Alkaline phosphatase (ALP) staining and Alizarin red staining were conducted to examine osteogenesis and mineralization, respectively. C1: control cells cultured in osteogenic medium without dexamethasone; C2: Control cells cultured in medium without osteogenic supplements. (C) MC3T3-E1 cells were cultured in osteogenic medium containing different doses of dexamethasone as indicated. Quantitative real-time PCR (qRTPCR) was performed at $3 \mathrm{~d}$ after treatment to measure mRNA expression of FUT8 as well as osteogenesis- and adipogenesis-related genes. ACTB was used as an internal reference. Data are expressed as the mean \pm standard deviation (SD). $* P<0.05, * * P<0.01, * * * P<0.001$ vs. 0 M. All experiments were independently repeated at least 3 times. Dex, Dexamethasone; FUT8, fucosyltransferase 8; ALP: alkaline phosphatase. 

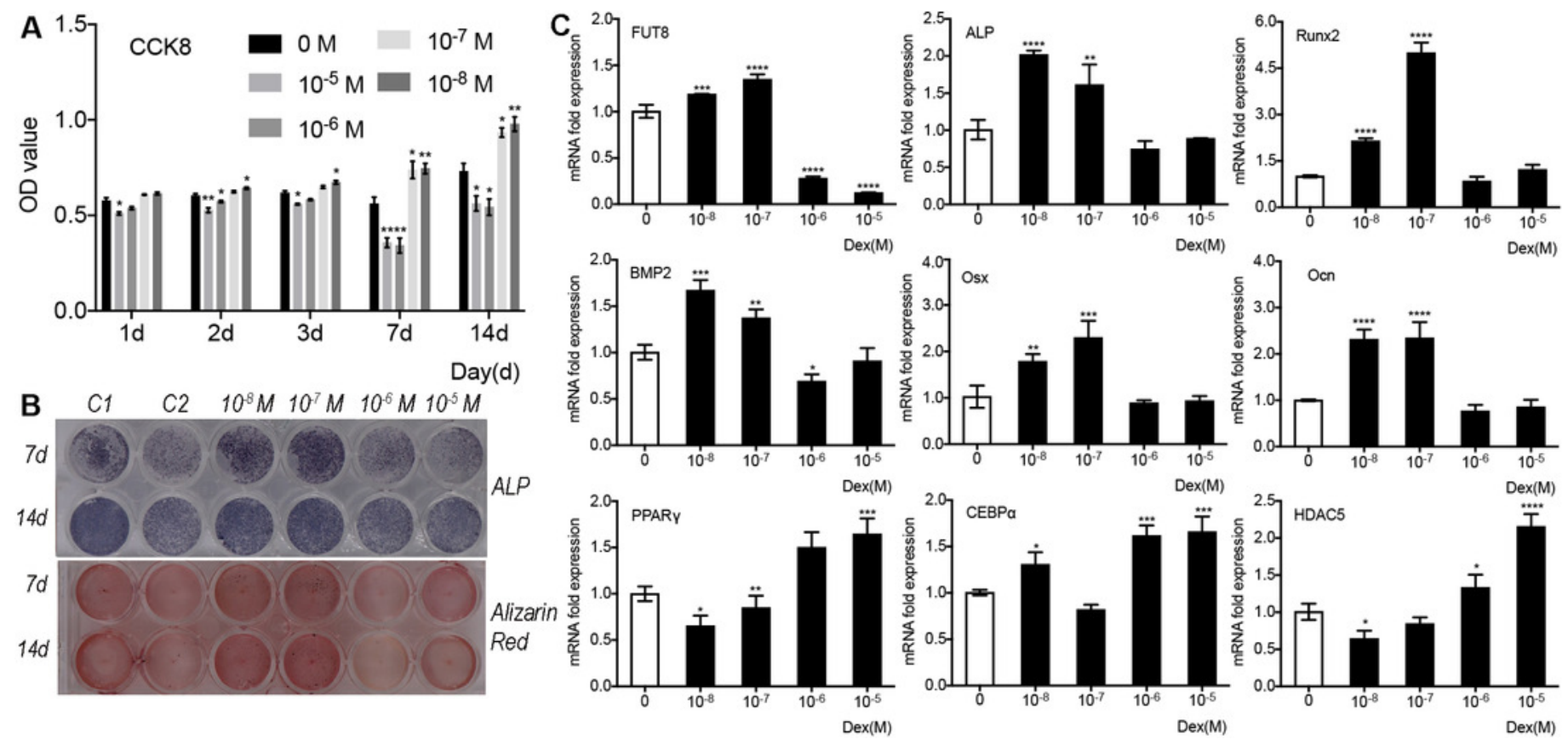


\section{Figure 2}

Figure 2

Figure 2. Overexpression of FUT8 promoted osteogenic differentiation of MC3T3-E1 cells regardless of the presence or absence of high-dose dexamethasone. MC3T3-EI cells were transiently transfected with empty or FUT8-overexpressing vectors and incubated in osteogenic medium in the absence or presence of high-dose dexamethasone for 5 or 14 days (A) ALP staining and Alizarin red staining were performed to examine osteogenesis and mineralization, respectively (B) qRT-PCR was performed at 3 days after treatment to measure mRNA expression of osteogenesis-related genes. ACTB was used as an internal reference. Data are expressed as the mean \pm SD. $* P<0.05,{ }^{*} * P<0.01, * * * P<0.001,{ }^{*} * * * P<0.0001$ vs. pEGFP-C1. All experiments were independently repeated at least 3 times. FUT8, fucosyltransferase; ALP, alkaline phosphatase; Dex, dexamethasone.
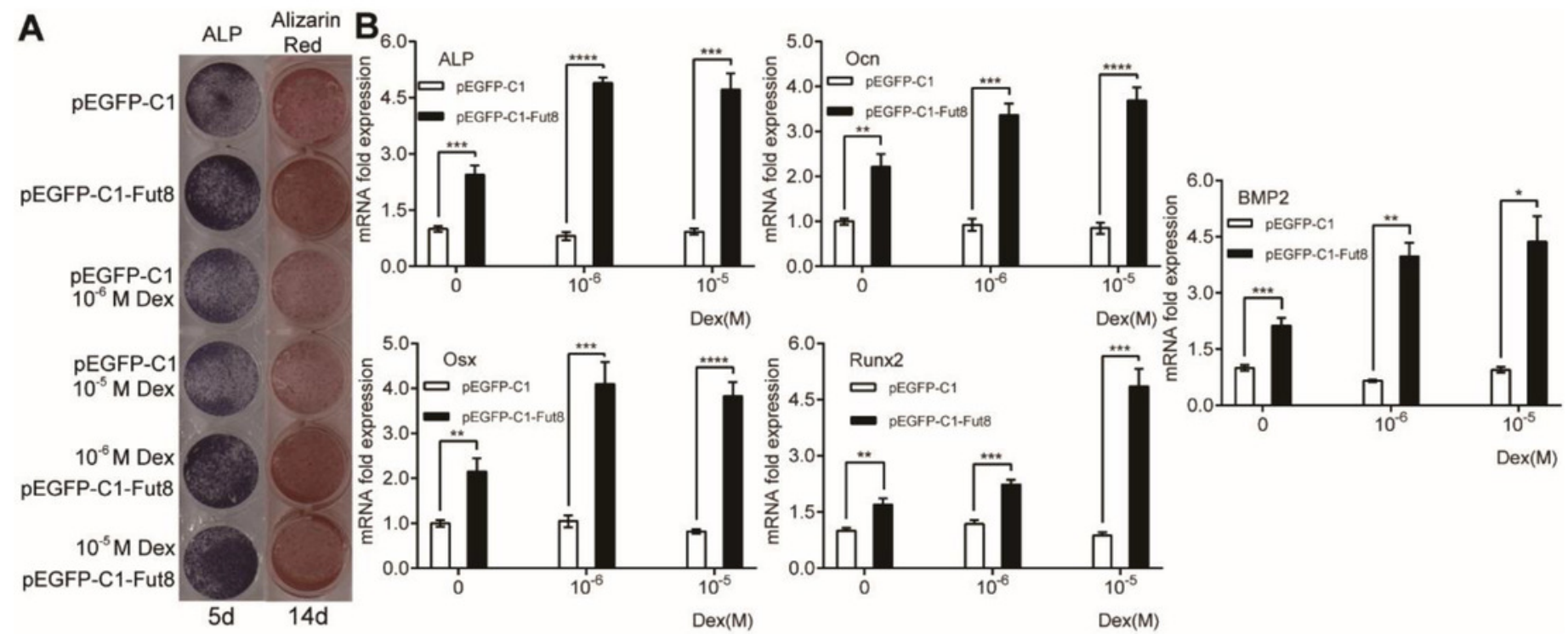


\section{Figure 3}

Figure 3

Figure 3. The effects of overexpression of FUT8 on TGF $\beta$ /Smad signaling in MC3T3-

E1 cells. MC3T3-E1 cells were transfected with empty or FUT8-overexpressing vectors and cultured in osteogenic medium containing different doses of dexamethasone $\left(0,10^{-6}\right.$, and $10^{-5}$ M) for 3 days. (A-C) qRT-PCR was performed to measure mRNA expression of the components of the TGF $\beta / S m a d$ pathway. ACTB was used as an internal reference. Data are expressed as the mean $\pm \mathrm{SD}$. $* P<0.05, * * P<0.01,{ }^{*} * * P<0.001, * * * * P<0.0001$ vs. pEGFP-

C1. (D) Western blot analysis was conducted to measure protein expression of phosphorylated and total Smad2/3 protein levels. $\beta$-actin was used as an internal reference. All experiments were independently repeated at least 3 times. FUT8, fucosyltransferase; Dex, dexamethasone.
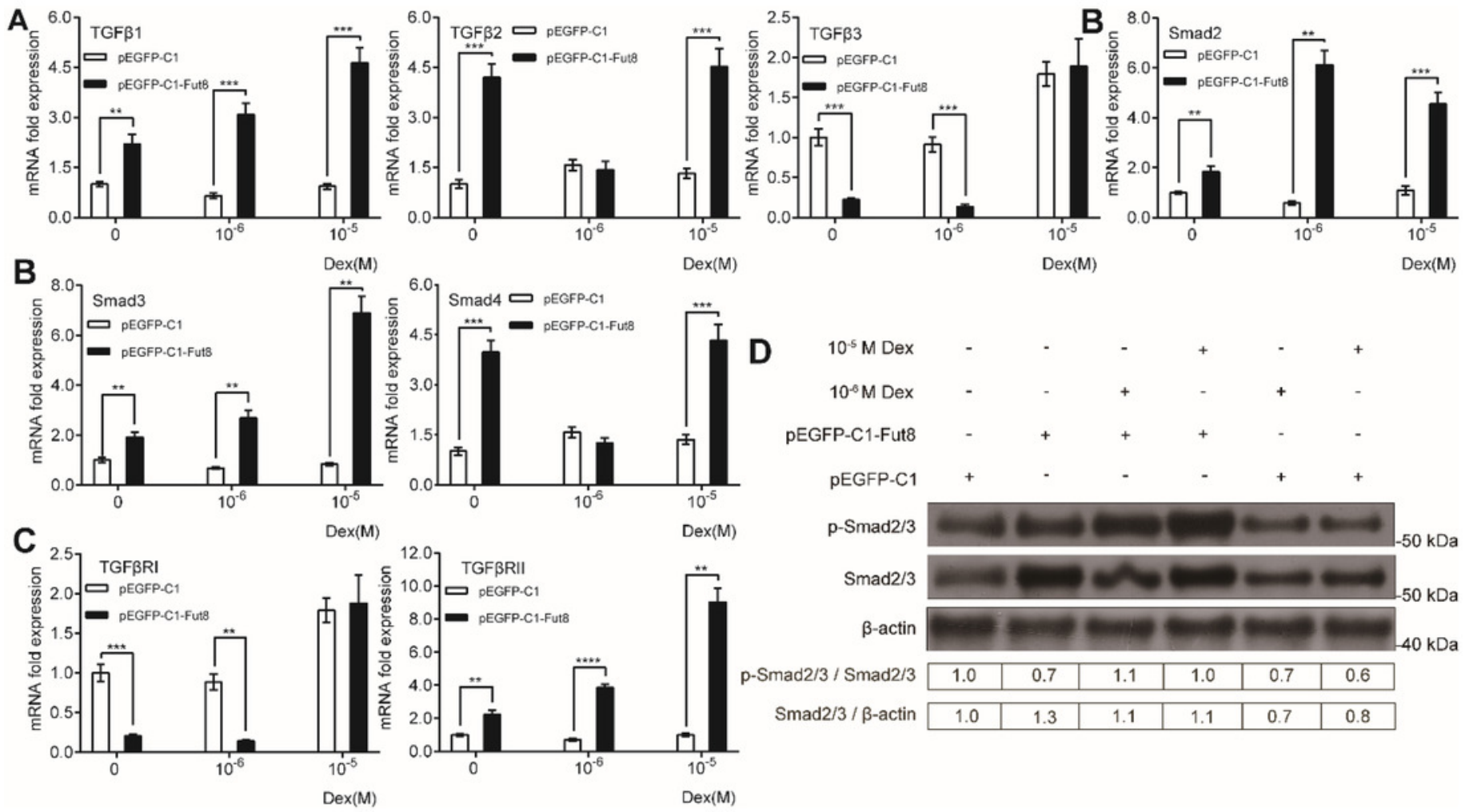

D $\quad 0^{1.5} \mathrm{M}$ Dex $10^{-6} \mathrm{M}$ Dex pEGFP-C1-Fut8 pEGFP-C1 p-Smad2/3

$$
\text { (M) }
$$

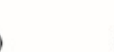




\section{Figure 4}

Figure 4

Figure 4. Overexpression of FUT8 promoted core fucosylation of TGFBRs and TGF$\beta 1$ binding to TGFBRs in MC3T3-E1 cells in the presence of high-dose

dexamethasone. MC3T3-E1 cells were transfected with empty or FUT8-overexpressing vectors and cultured in osteogenic medium containing different doses of dexamethasone $(0$, $10^{-6}$, and $10^{-5} \mathrm{M}$ ) as indicated for 3 days. (A) Lens culinaris agglutinin (LCA)-based immunofluorescence staining was performed to detect $\alpha 1,6$-fucosylated trimannose-core structure in MC3T3-E1 cells. Scale bar $=100 \mu \mathrm{m}$. (B) LCA blot analysis was performed to determine the level of core fucosylated-proteins. (C) Co-immunoprecipitation was conducted to measure the levels of core fucosylated-TGFBR1 and TGFBR2. (D) MC3T3-E1 cells (from A) were transfected with empty or His-tagged TGF- $\beta 1$ (His-TGF- $\beta 1$ )-overexpressing vectors. The conditioned medium was collected at 2 days after transfection. MC3T3-E1 cells were cultured in the conditioned medium for $4 \mathrm{~h}$, and the cell lysates were collected. The His-TGF- $\beta 1$ protein bound to endogenous TGFBRs were detected by the ALP-conjugated anti-His antibody and quantified by measuring the bound ALP using a p-nitrophenyl phosphate substrate. Data are expressed as the mean $\pm \mathrm{SD}$. $* P<0.05$, $* * P<0.01$, *** $P<0.001$, **** $P<0.0001$ vs. empty vector. mOD405, milli-absorbance units at $405 \mathrm{~nm}$. All experiments were independently repeated at least 3 times. 


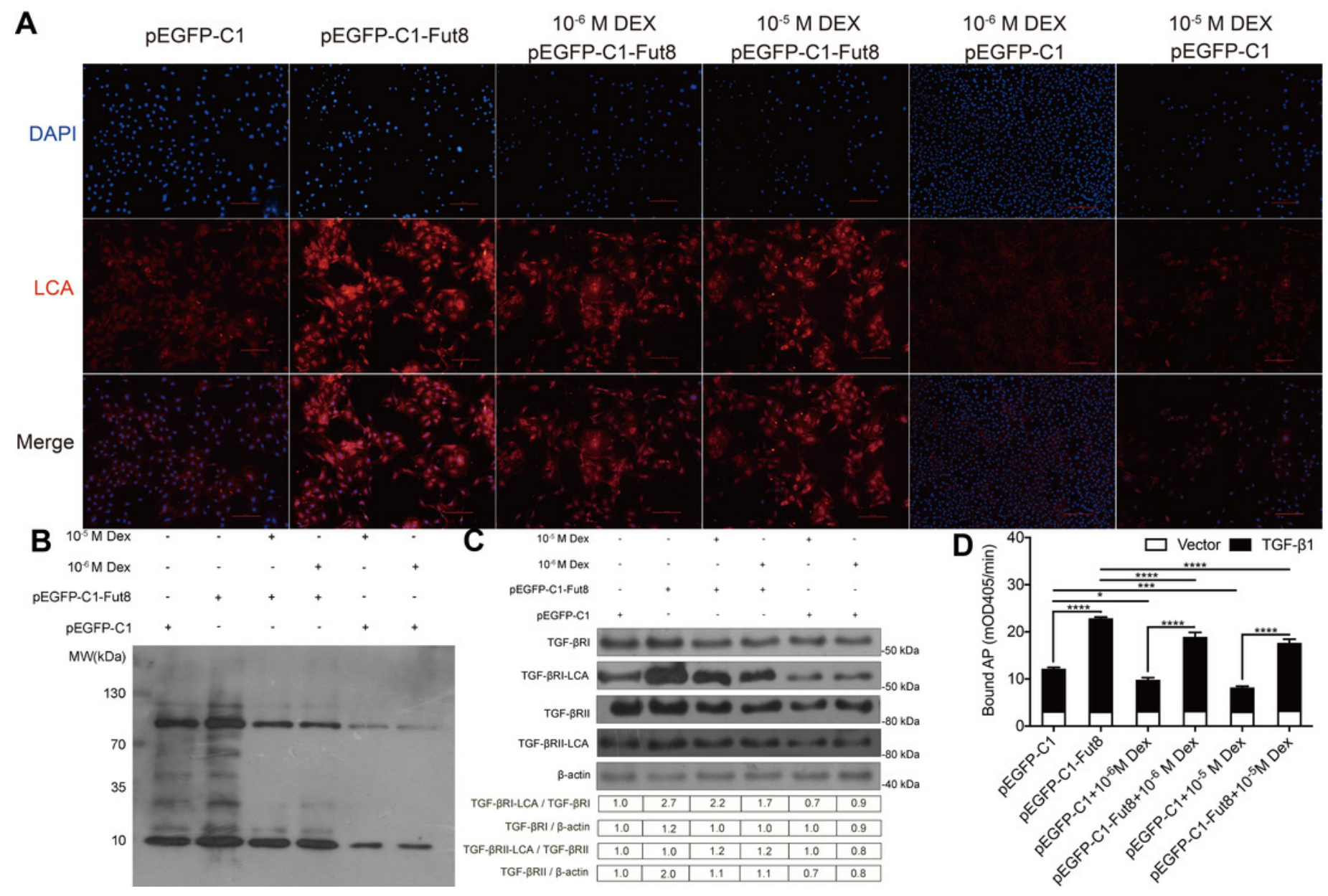

